University of Wollongong

Research Online

Australian Institute for Innovative Materials -

Papers

Australian Institute for Innovative Materials

$1-1-2018$

\title{
Field dependence of the ferromagnetic/superconducting proximity effect in a YBCO/STO/LCMO multilayer
}

Oliver Paull

University of Wollongong, ohcp627@uowmail.edu.au

Alexey Pan

University of Wollongong, pan@uow.edu.au

Grace Causer

University of Wollongong, glc052@uowmail.edu.au

Sergey Fedoseev

University of Wollongong, sergey@uow.edu.au

Antony Jones

University of Wollongong, anj093@uowmail.edu.au

See next page for additional authors

Follow this and additional works at: https://ro.uow.edu.au/aiimpapers

Part of the Engineering Commons, and the Physical Sciences and Mathematics Commons

Research Online is the open access institutional repository for the University of Wollongong. For further information contact the UOW Library: research-pubs@uow.edu.au 


\title{
Field dependence of the ferromagnetic/superconducting proximity effect in a YBCO/STO/LCMO multilayer
}

\author{
Abstract \\ The interaction between superconductivity and magnetism in spatially confined heterostructures of thin \\ film multilayers is investigated in the ferromagnetic manganite La2/3Ca1/3MnO3 (LCMO) and the high- \\ temperature superconductor YBa2Cu307- $\delta$ (YBCO) mediated by an intermediate insulating SrTiO3 (STO) \\ layer. The STO layer is used to mediate and tune the range of interactions between the ferromagnet and \\ superconductor. A magnetically depleted layer with zero-magnetisation within the LCMO layer is shown by \\ polarised neutron reflectometry measurements. This zero-magnetisation layer is caused by the onset of \\ superconductivity in YBCO despite being separated by an insulating layer with a thickness much larger \\ than the superconducting coherence length. The magnetic field dependence of this interaction is also \\ explored. We show that the magnetism of the depleted layer can be restored by applying a magnetic field \\ that partially destroys the superconductivity in YBCO, restricting the electronic interaction between the \\ materials. \\ Disciplines \\ Engineering | Physical Sciences and Mathematics

\section{Publication Details} \\ Paull, O. H. C., Pan, A. V., Causer, G. L., Fedoseev, S. A., Jones, A., Liu, X., Rosenfeld, A. \& Klose, F. (2018). \\ Field dependence of the ferromagnetic/superconducting proximity effect in a YBCO/STO/LCMO \\ multilayer. Nanoscale, 10 (40), 18995-19003.

\section{Authors} \\ Oliver Paull, Alexey Pan, Grace Causer, Sergey Fedoseev, Antony Jones, Xinzhi Liu, Anatoly B. Rosenfeld, \\ and Frank Klose
}



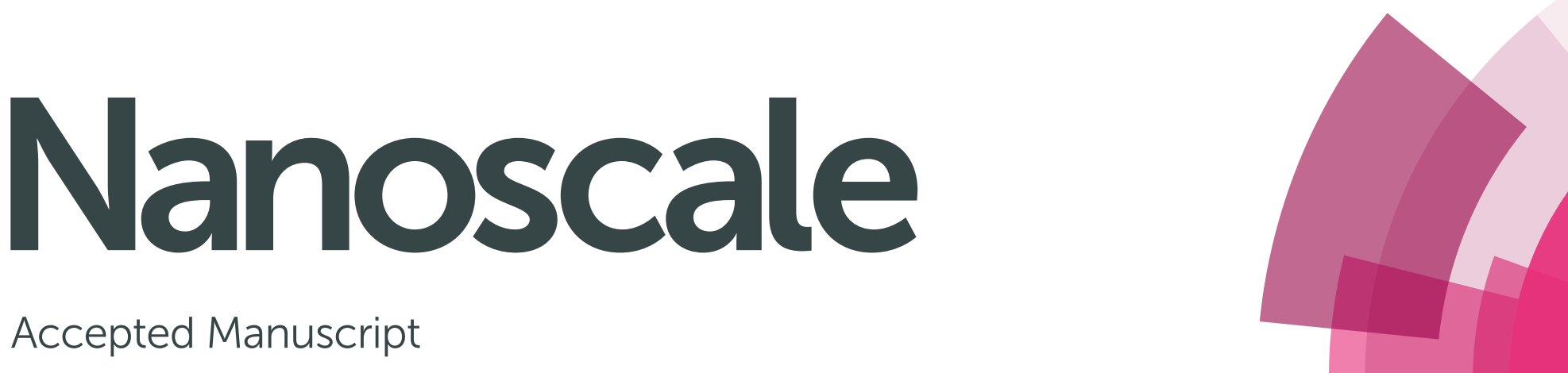

\section{Accepted Manuscript}

This article can be cited before page numbers have been issued, to do this please use: O. Paull, A. V. Pan, G. L. Causer, S. A. Fedoseev, A. Jones, X. Liu, A. Rozenfeld and F. Klose, Nanoscale, 2018, DOI:
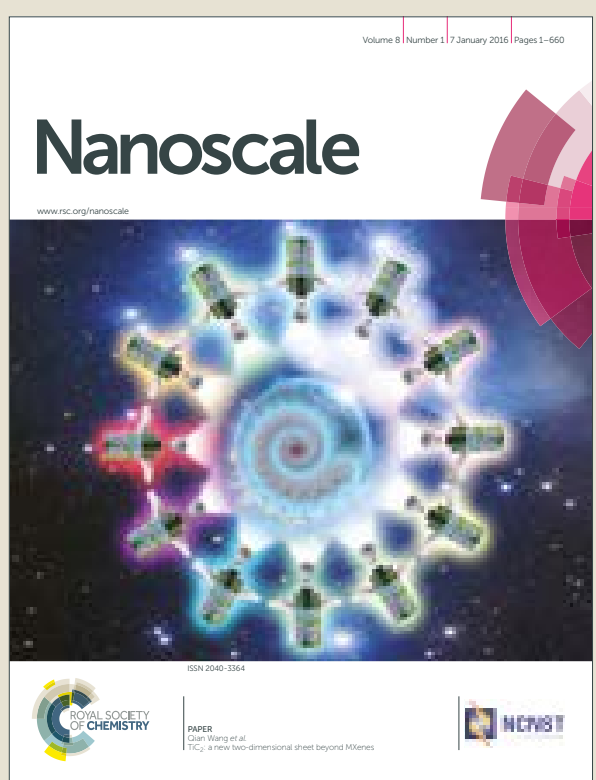

This is an Accepted Manuscript, which has been through the Royal Society of Chemistry peer review process and has been accepted for publication.

Accepted Manuscripts are published online shortly after acceptance, before technical editing, formatting and proof reading. Using this free service, authors can make their results available to the community, in citable form, before we publish the edited article. We will replace this Accepted Manuscript with the edited and formatted Advance Article as soon as it is available.

You can find more information about Accepted Manuscripts in the author guidelines.

Please note that technical editing may introduce minor changes to the text and/or graphics, which may alter content. The journal's standard Terms \& Conditions and the ethical guidelines, outlined in our author and reviewer resource centre, still apply. In no event shall the Royal Society of Chemistry be held responsible for any errors or omissions in this Accepted Manuscript or any consequences arising from the use of any information it contains. 


\title{
Field Dependence of the Ferromagnetic/Superconducting Proximity Effect in a YBCO/STO/LCMO Multilayer
}

\author{
Oliver H. C. Paull ${ }^{a, b},{ }^{*}$ Alexey V. Pan ${ }^{a, c},^{\dagger}$ and Grace L. Causer ${ }^{a, b}$, Sergey A. \\ Fedoseev $^{a, d}$, Antony Jones ${ }^{a, e}$, Xinzhi Liu ${ }^{b, f}$, Anatoly Rosenfeld ${ }^{d}$, Frank Klose ${ }^{b \ddagger}$ \\ ${ }^{a}$ Institute for Superconducting and Electronic Materials (ISEM), \\ University of Wollongong, Northfields Avenue, Wollongong, NSW 2522, Australia \\ ${ }^{b}$ Australian Nuclear Science and Technology Organisation (ANSTO), Lucas Heights 2234, Australia \\ ${ }^{c}$ National Research Nuclear University MEPhI (Moscow Engineering Physics Institute), \\ 31 Kashirskoye Shosse, 115409, Moscow, Russian Federation \\ ${ }^{d}$ Centre for Medical Radiation Physics, University of Wollongong, \\ Northfields Avenue, Wollongong, NSW 2522, Australia \\ ${ }^{e}$ CSIRO Manufacturing, Bradfield Rd, Lindfield NSW, Australia \\ ${ }^{f}$ Helmholtz-Zentrum-Berlin für Materialien und Energie, \\ Hahn-Meitner-Platz 1, D-14109 Berlin, Germany
}

(Dated: 29 March 2018)

The interaction between superconductivity and magnetism in spatially confined heterostructures of thin film multilayers is investigated in the ferromagnetic manganite $\mathrm{La}_{2 / 3} \mathrm{Ca}_{1 / 3} \mathrm{MnO}_{3}$ (LCMO) and the high-temperature superconductor $\mathrm{YBa}_{2} \mathrm{Cu}_{3} \mathrm{O}_{7-\delta}$ (YBCO) mediated by an intermediate insulating $\mathrm{SrTiO}_{3}$ (STO) layer. The STO layer is used to mediate and tune the range of interactions between the ferromagnet and superconductor. A magnetically depleted layer with zeromagnetisation within the LCMO layer is shown by polarised neutron reflectometry measurements. This zero-magnetisation layer is caused by the onset of superconductivity in YBCO despite being separated by an insulating layer with a thickness much larger than the superconducting coherence length. The magnetic field dependence of this interaction is also explored. We show that the magnetism of the depleted layer can be restored by applying a magnetic field that partially destroys the superconductivity in YBCO, restricting the electronic interaction between the materials. 
* Corresponding author's email: ohcp627@uowmail.edu.au

† Corresponding author's email: alexey_pan@uow.edu.au

$\ddagger$ Current address: Guangdong Technion Israel Institute of Technology, Shantou, Guangdong 515063, PR

China, email: frank.klose@gtiit.edu.cn 


\section{INTRODUCTION}

The interaction between superconductivity and ferromagnetism as two mutually exclusive order parameters coexisting is an intense topic of research at present. This is attributed to a number of recent experimental findings such as orbital reconstruction at the interface of $\mathrm{YBa}_{2} \mathrm{Cu}_{3} \mathrm{O}_{7-\delta}(\mathrm{YBCO}) / \mathrm{La}_{2 / 3} \mathrm{Ca}_{1 / 3} \mathrm{MnO}_{3}$ (LCMO) hybrid structures [1], long range chargedensity-wave proximity effects [2], as well as a magnetic proximity effect in YBCO/LCMO superlattices [3-6]. A thorough understanding of the interactions occurring at the interface between these two exotic materials has the potential to pave the way to a new era in electronics and devices [7].

A magnetically depleted (MD) layer was observed in YBCO/LCMO superlattices within the ferromagnetic material adjacent to the interface[3]. This MD layer had a reduced or completely suppressed magnetisation as observed by polarised neutron reflectometry (PNR) and resonant X-ray techniques. Self-consistent total energy calculations of this superlattice structure within density functional theory also confirmed that the coupling between Mn atoms becomes very weak and possibly anti-ferromagnetic near the interface [6]. Note, in contrast to the present work, these results were obtained for the interfaces between superconducting and ferromagnetic materials having no intermediate layers, which could potentially attenuate and/or control the interactions.

The range of influence of LCMO on YBCO was approximated by Fedoseev et al. by introducing an insulating $\mathrm{SrTiO}_{3}$ (STO) layer between the ferromagnet and superconductor $[8,9]$. It was found that the proximity effect of a $150 \mathrm{~nm}$ thick LCMO layer affecting the critical temperature of a $200 \mathrm{~nm}$ YBCO layer became negligible for STO thicknesses of $>5 \mathrm{~nm}$.

Trilayer YBCO/STO/LCMO films, consisting of $30 \mathrm{~nm}$ LCMO and YBCO layers separated by varying thicknesses of STO, were investigated using PNR by Prajapat et al. [10, 11]. It was observed that even with the presence of a thin insulating STO layer $(2.5$ and $5 \mathrm{~nm})$ between the superconducting and ferromagnetic material, a MD layer was still present in LCMO. This result was exclusively found below the superconducting transition temperature $\left(T_{c}\right)$, where it was shown that the width of the MD layer was inversely proportional to the thickness of the STO interlayer. Although, the superconducting properties of the YBCO layers were relatively weak as indicated by a reduced $T_{c}[10,11]$, the tunnelling of Cooper pairs 
through the STO layer was suggested to be responsible for the suppressed magnetisation in the LCMO layer near the interface.

In this work, we investigate the magnetic field dependence of the magnetically depleted layer in YBCO $(30 \mathrm{~nm}) / \mathrm{STO}(1.5 \mathrm{~nm}) /$ LCMO $(30 \mathrm{~nm})$ trilayers. We show that the application of a magnetic field results in an increase of the magnetisation in LCMO at the interface with STO, as seen by PNR investigations from the reversal of magnetisation suppression in the depleted layer. This reversal may indicate another tool, along with the intermediate insulating layer, for the manipulation and control of the confined magnetic properties in superconducting/ferromagnetic heterostructures, for their potential use in future spintronic applications.

\section{RESULTS}

\section{A. X-Ray Diffraction}

In this work, we investigate the YBCO/STO/LCMO trilayers of $30 \mathrm{~nm} / 1.5 \mathrm{~nm} / 30 \mathrm{~nm}$ thick grown by pulsed laser deposition (PLD) on an Yttria-Stabilised Zirconia (YSZ) substrate as described in our previous work $[15,16]$.

$\mathrm{X}$-Ray Diffraction (XRD) is used to indicate the quality of the epitaxial growth of the sample. Figure 1 shows that all diffraction peaks measured in the $\omega-2 \theta$ geometry are $c$-axis oriented demonstrating epitaxial growth of the sample in the correct crystallographic direction. The observation of YBCO peaks up to (00 10) shows the high quality growth of the superconducting material in the trilayer.

The $\mathrm{YBCO}(001)$ diffraction peak shown in the inset to Figure 1 (not visible in the main figure) was fitted with the one-dimensional interference function $[\sin (N Q c) / \sin (Q c)]^{2}$. This equation describes the finite thickness interference fringes surrounding a diffraction peak, and the thickness of a single-crystal layer can be extracted from it. In this equation, $c$ is the $c$-axis lattice parameter, $Q$ is the momentum transfer proportional to the experimental reflection angle, and $N$ is the number of unit cells in the $z$-direction. The fitting procedure has determined $N=28$ and $c=11.74 \AA$. $N$ determines the thickness of the YBCO layer which agrees with the thickness found by both XRR and PNR measurements, and the measured $c$-axis parameter for YBCO is elongated by $0.06 \AA$ from its bulk value $[12,13]$ 


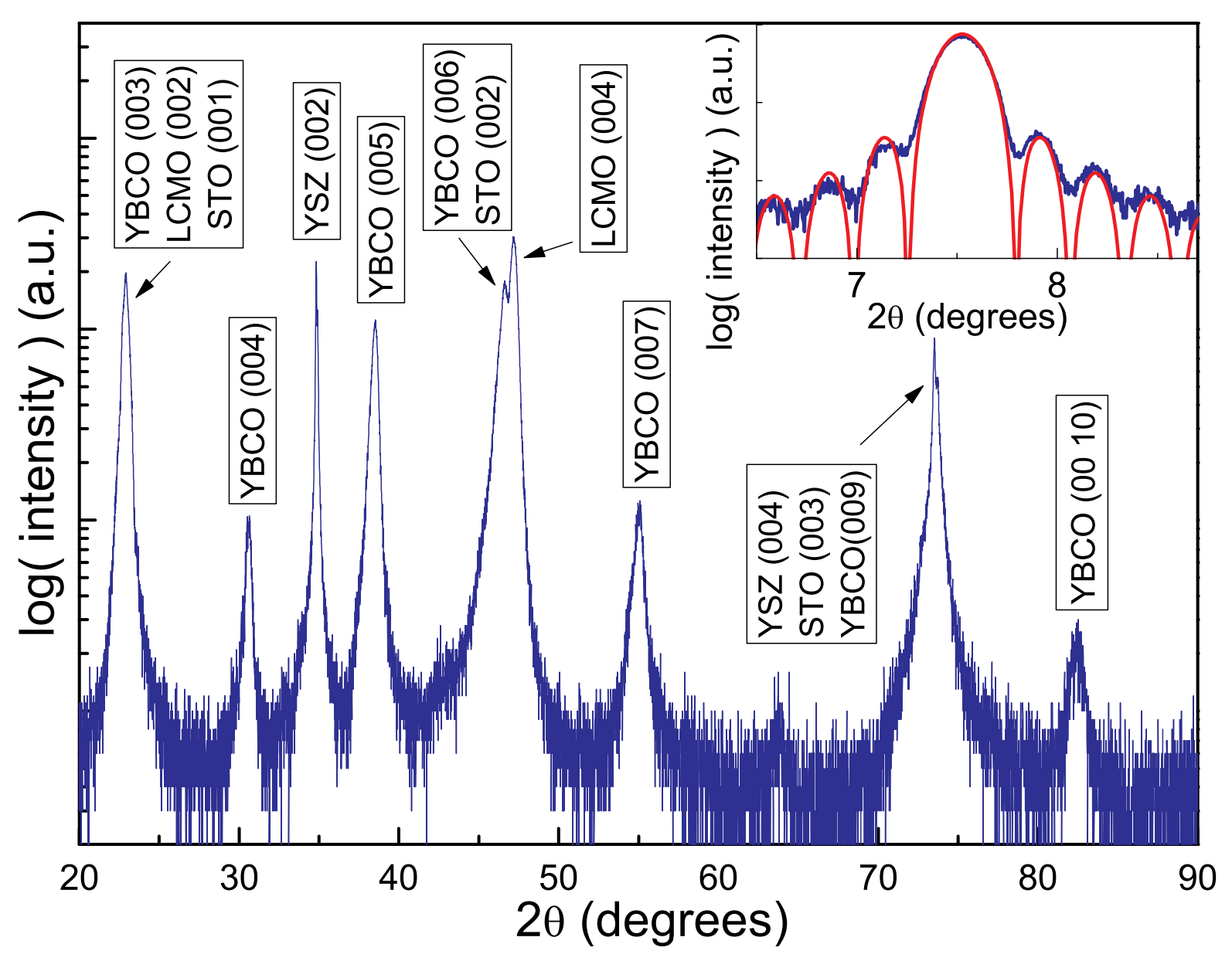

FIG. 1. X-ray Diffraction measurements in $\omega-2 \theta$ geometry. Inset shows the YBCO (001) peak fitted with the 1D interference function.

indicating an in-plane compressive strain on YBCO from the YSZ substrate.

In order to further quantify the quality of the film's uniaxial growth, rocking curve measurements were performed on YBCO and LCMO diffraction peaks (results not shown). The full width at half maximum (FWHM) extracted from a Gaussian fit of the rocking curve around the YBCO (005) and LCMO (004) reflections are $\Delta \omega=0.397^{\circ}$ and $\Delta \omega=0.371^{\circ}$ respectively, with an instrumental resolution of $0.031^{\circ}$. These values verify the low mosaicity of the YBCO and LCMO layers in the multilayer. 


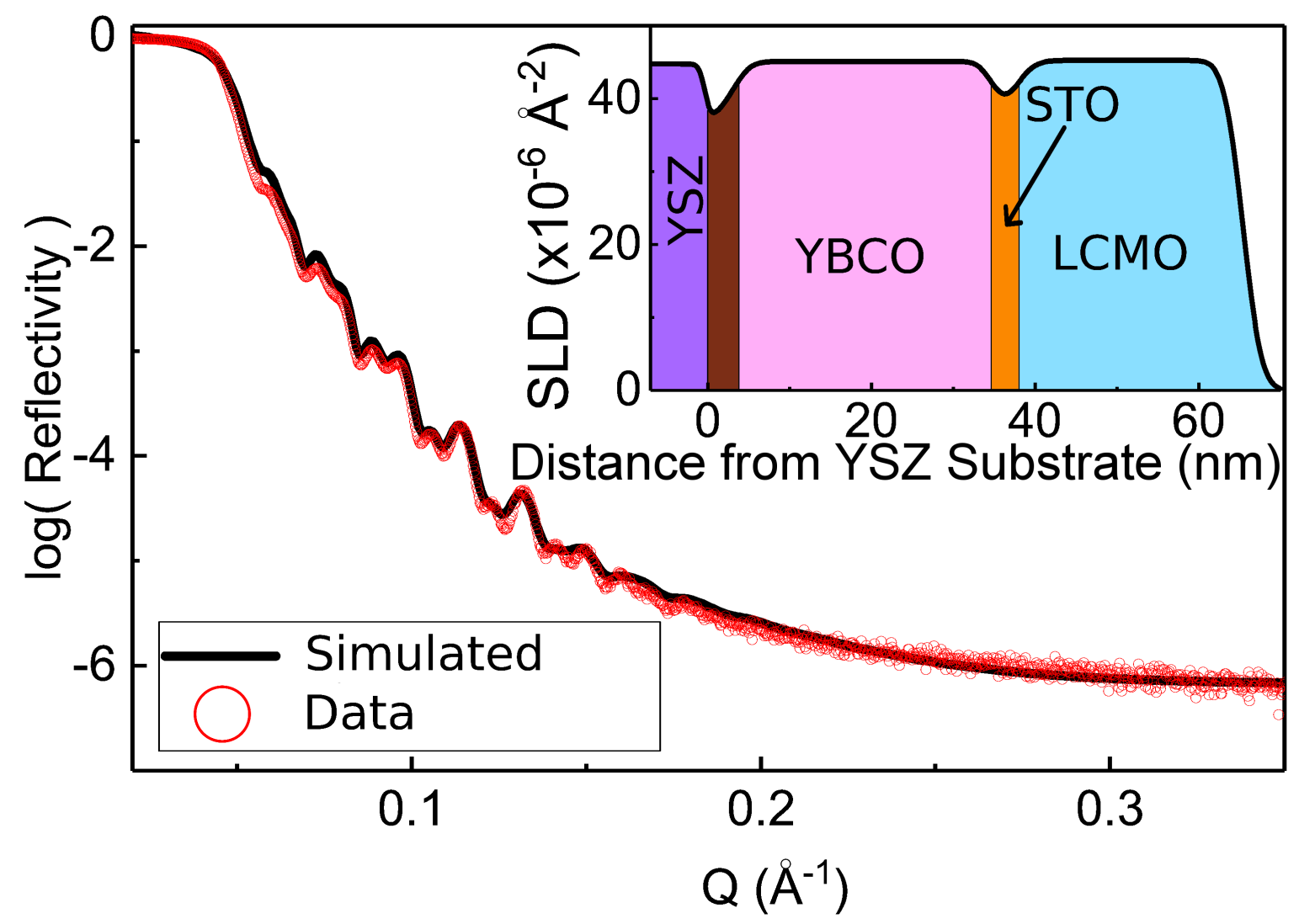

FIG. 2. X-ray reflectometry of trilayer. The inset shows the corresponding electron SLD profile. The first two layers on the left-hand side of the SLD profile inset are the substrate YSZ and a layer of initial imperfect YBCO growth.

\section{B. X-ray Reflectometry}

Figure 2 displays the X-ray reflectometry data (circle symbols) and fit (line) for the YBCO/STO/LCMO trilayer. The scattering length density (SLD) profile constructed from fitting the X-ray reflectometry data is shown in the inset of Figure 2. The SLD profile shows an approximate thickness of the layers, agreeing with the $\sim 32 \mathrm{~nm}$ thickness of YBCO and LCMO determined by the interference function and by the pulsed laser deposition parameters. The roughness in the sample is less than $2 \mathrm{~nm}$ on the surface and less than $1 \mathrm{~nm}$ throughout the material layers.

The dip in SLD between the substrate and YBCO is a layer of initial non-perfect epitaxial 

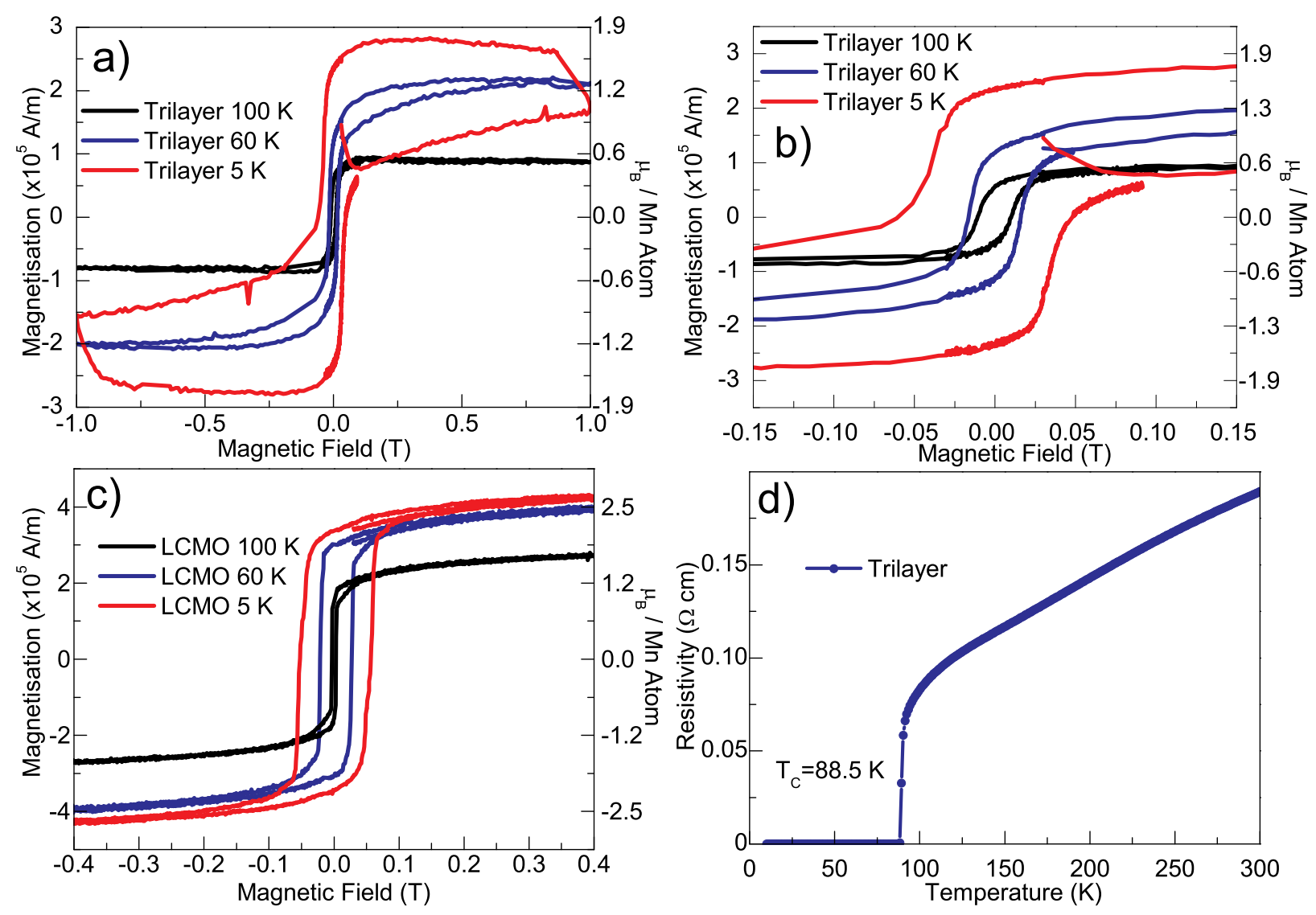

FIG. 3. (a) In-plane magnetisation hysteresis curves of the trilayer at different temperatures. (b) Low-field region of the hysteresis curves of the trilayer shown in a). (c) In-plane magnetisation curve of LCMO thin film, and (d) Resistivity, indicating a sharp superconducting transition at $T_{c}=88.5 \mathrm{~K}$.

growth of YBCO on YSZ before proper growth starts. This non-perfect layer is usually present in PLD grown films on any substrate (e.g., see transmission electron microscopy images near the substrate in Refs. $[14,15])$. This feature was also found in the PNR SLD profile, but it did not affect the measurements in any other way. The X-ray diffraction in Figure 1 also shows that this initial layer does not produce any additional diffraction peaks, confirming that there is no other crystallographic phases present. 


\section{Magnetometry and Transport Measurements}

Magnetic hysteresis measurements have been carried out at $100 \mathrm{~K}, 60 \mathrm{~K}$, and $5 \mathrm{~K}$. Each measurement is obtained by field-cooling the sample in the magnetic field of $B_{a}=0.03 \mathrm{~T}$ applied in-plane to the trilayer sample, then starting the field sweep from that magnetic field. The magnetisation loops are shown in Figure 3a) with Figure 3b) displaying the low-field part of the curves.

Transport measurements shown in Figure 3d) display a superconducting critical temperature $T_{c}=88.5 \mathrm{~K}$ with a sharp transition. This value of $T_{c}$ is remarkably close to the optimal value of $91 \mathrm{~K}$ for YBCO thin films $[14,16]$, especially considering that (i) the thickness of the YBCO film in this work is only $30 \mathrm{~nm}$ (YBCO film thicknesses below $\sim 50 \mathrm{~nm}$ are known to exhibit lower $T_{c}$ due to reduced crystallinity at the interface with a substrate upon the initial epitaxial growth [17]); and that (ii) it is in close proximity to the ferromagnetic material, which might affect the YBCO film via residual stray fields. However, we would like to emphasise that there is an STO interlayer between the YBCO and LCMO layers [8, 9], presumably preventing close range interactions, which would suppress superconductivity and $T_{c}$ due to the inverse proximity effect $[18,19]$. On the other hand, we do not expect any measurable change in the "global" resistivity of the YBCO layer as the result of the loss of Cooper pairs to the neighboring STO and LCMO layers due to the proximity effect. For this, a localised technique such as scanning tunnelling spectroscopy [18, 19] should be employed.

This $T_{c}$ value of $88.5 \mathrm{~K}$ is significantly higher than what was found in similar hybrid systems in the literature $[5,10,11]$. This is further evidence of the high-quality crystallinity in our YBCO thin film layer.

At $T=100 \mathrm{~K}$, which is above $T_{c}$, the hysteresis loops shows purely ferromagnetic behaviour (Fig. 3a,b) similar to that measured for the pure LCMO film (Fig. 3c). As the temperature is reduced below $T_{c}$ to $60 \mathrm{~K}$ and $5 \mathrm{~K}$, the loops become increasingly distorted by addition of the irreversible component of superconducting magnetisation over the broad field range due to pinning properties in the YBCO layer. A similar superposition of ferromagnetic and superconducting magnetisation response was previously observed in different hybrid structures [20, 21].

The virgin part of the magnetisation loop at $5 \mathrm{~K}$ initially exhibits a typical behaviour for 
the initial magnetic flux penetration in a type II superconductor above the first critical field (Fig. 3a,b). Notably, the magnetisation remains positive for the entire virgin curve due to the superposition of the saturated magnetisation of ferromagnetic LCMO and the magnetic flux frozen in YBCO after the field-cooling procedure.

Thus, it is evident that the trilayer at $T=5 \mathrm{~K}$ and $60 \mathrm{~K}$ displays strong contributions from both ferromagnetism in LCMO as well as superconductivity in YBCO.

\section{Polarised Neutron Reflectometry}

Polarised neutron reflectometry (PNR) was measured for the sample in two different magnetic fields (0.03 T and $1 \mathrm{~T}$ ) for three different temperatures: $100 \mathrm{~K}$ (Fig. 4), and $60 \mathrm{~K}$ and $5 \mathrm{~K}$ (Fig. 5a-c). Each measurement was obtained by field-cooling the sample in $B_{a}=0.03 \mathrm{~T}$ applied in-plane to the trilayer down to the desired temperature, then changing the magnetic field to the value needed.

Each fitted reflectivity model corresponds to an associated SLD profile. From the reflectivities of the $\mathrm{R}^{++}$and $\mathrm{R}^{--}$spin channels, the normalised spin asymmetry (NSA) can be found. This is defined as

$$
N S A=\frac{R^{++}-R^{--}}{R^{++}+R^{--}}
$$

It is sensitive exclusively to the magnetic features of the sample, which will be used to help determine the magnetic profile throughout the sample.

The PNR measurement at $100 \mathrm{~K}$ (above $T_{c}$ for $\mathrm{YBCO}$ ) in $0.03 \mathrm{~T}$ (Fig. 4a) has been fitted using Simulreflec simulation software [22]. The corresponding SLD profile in Figure 4c) displays a magnetic SLD (MSLD) profile (red) within the LCMO layer that persists up to the LCMO/STO interface. As these data are measured above $T_{c}$ for YBCO, they serve as the undisturbed benchmark for the LCMO magnetisation depth profile.

This magnetic SLD has also been given a second $y$-axis in Fig. 4c) corresponding to the number of Bohr magnetons per Mn atom.

The nuclear SLD of LCMO (black bold line) in Fig. 4c) is reduced as it approaches the air interface. In this region, the magnetisation (red) is also reduced compared to the middle of the layer, which is consistent with previous neutron studies on doped manganites [23]. The LCMO layer also has an increased nuclear SLD towards the LCMO/STO interface, accompanied with a reduced magnetisation in comparison to the middle of the layer. It 

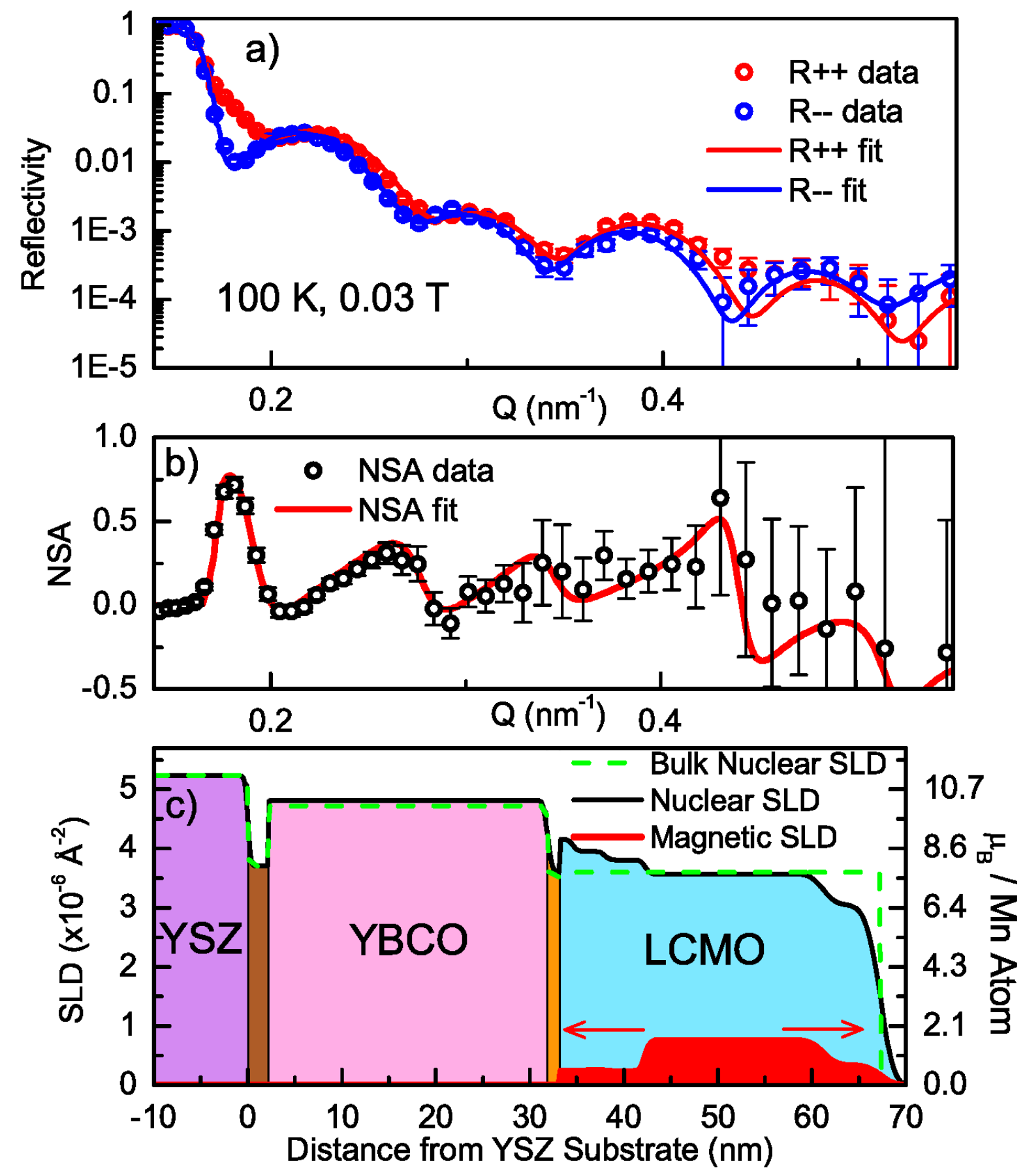

FIG. 4. (a) Polarised neutron reflectometry data (symbols) and fitted model (lines) at $T=100 \mathrm{~K}$ and $B_{a}=0.03 \mathrm{~T}$, (b) Corresponding NSA data (symbols) and fit (line), and (c) constructed SLD profile based on fitted model in (a). The right $y$-axis in (c) translates the MSLD to Bohr magnetons per Mn atom. 
has previously been found that LCMO deposited on STO causes tensile strain, which in turn proliferates oxygen vacancies in LCMO near the interface. These oxygen vacancies significantly reduce the magnetisation [24]. The reduced nuclear SLD near the LCMOair interface is indicative of relaxation in the crystal lattice. The increased nuclear SLD in LCMO towards the LCMO/STO interface is understood to be as a result of YBCO epitaxial growth. YBCO films develop by the island growth model (Stranski-Krastanov mode), and as such their surfaces are not atomically flat $[13,25]$. Deposition of STO and LCMO on top of a YBCO structure such as this propagates the waviness throughout the multilayer in a similar fashion as observed for superlattices in ours and others' previous work [15, 26]. The resultant laterally averaged SLD is the weighted addition of mainly LCMO and YBCO, which has a net increase in structural SLD compared to bulk LCMO.

It is worthwhile to once again emphasise that in the absence of the superconducting state in the YBCO layer, the magnetic (red) SLD profile of LCMO extends all the way up to the STO layer at $B_{a}=0.03 \mathrm{~T}$ and $T=100 \mathrm{~K}$, which is well above $T_{c}=88.5 \mathrm{~K}$ for our $\mathrm{YBCO}$ layer.

Figure 5 shows the low-field $(0.03 \mathrm{~T})$ and high-field $(1 \mathrm{~T})$ PNR measurements for $5 \mathrm{~K}$ and $60 \mathrm{~K}$ for the trilayer sample. The inset of each measurement is the associated MSLD for the data (red).

At $T<T_{c}$, there exists a $\sim 1.5 \mathrm{~nm}$ thick layer in the LCMO adjacent to the LCMO/STO interface that has approximately zero net magnetic moment in the applied field of $0.03 \mathrm{~T}$. This layer is shown in the MSLD profile inset in Figures 5a) and 5c). This suppression of the net magnetisation near the LCMO/STO interface is obviously triggered by the presence of superconductivity in the YBCO layer. Indeed, at $T>T_{c}$, the non-zero magnetisation extends up to STO/LCMO interface (Fig. 4c).

Under the application of the strong magnetic field of $1 \mathrm{~T}$, the layer of depleted magnetisation is restored (Figs. 5b and d). This is assumed to result from the partial destruction of the superconducting state by the high magnetic field, restricting the amount of Cooper pairs that are able to tunnel through (or be transported via) the STO layer to the LCMO layer. These pairs, once in the LCMO layer, would fall apart thereby reducing the number of the unpaired spin-polarised electrons around the $\mathrm{Mn}^{3+}$ in the LCMO layer, which are responsible for the double-exchange mechanism giving rise to ferromagnetism in the LCMO layer [27, 28]. The partial destruction of superconductivity is obvious from the reduced 

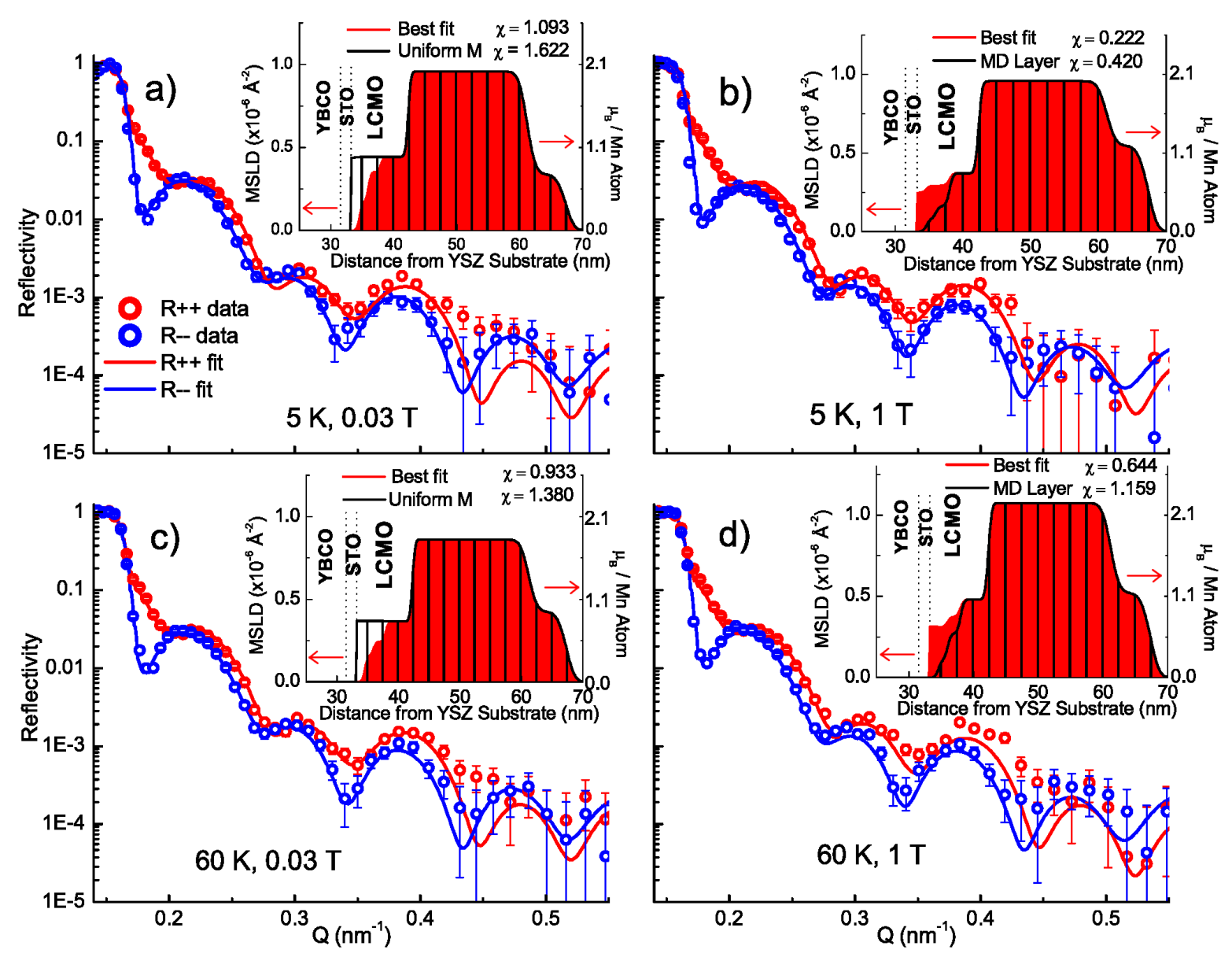

FIG. 5. Polarised neutron reflectivity at (a) $5 \mathrm{~K}$ in $0.03 \mathrm{~T}$, (b) $5 \mathrm{~K}$ in $1 \mathrm{~T}$, (c) $60 \mathrm{~K}$ in $0.03 \mathrm{~T}$, and (d) $60 \mathrm{~K}$ in $1 \mathrm{~T}$. The inset in each figure is the associated magnetic SLD for the PNR data. In all insets, the red area indicates the 'Best Fit' of the MSLD determined by the fitting process. The horizontal black lines (from $32 \mathrm{~nm}$ to $42 \mathrm{~nm}$ ) in a) and c) (measured at $0.03 \mathrm{~T}$ ) show a magnetic "Uniform M" model in which a constant LCMO magnetisation extends all the way to the STO interface. The black lines in b) and d) (measured at $1 \mathrm{~T}$ ) show a magnetically depleted "MD Layer" close to the STO interface. The black lines in all insets serve as alternate models for comparison to determine the presence of a magnetically depleted layer, and the vertical black stripes indicate that these models only vary from the "Best Fit" close to the interface. Each MSLD has been converted to Bohr magnetons per Mn atom on the right $\mathrm{y}$-axis. The $\chi$ parameter for each model is a statistical goodness-of-fit parameter for the normalised spin asymmetry. The interfaces between the LCMO, STO and YBCO layers are indicated by vertical dashed lines. 
height of the magnetisation loops at $B_{a}=1 \mathrm{~T}$ compared to the low-field region at $\sim 0.03 \mathrm{~T}$ in Fig. 3a), which is measured in the identical geometry employed for the PNR measurements (Fig. 5). Indeed, more vortices appear inside the superconductor with increasing field. This implies an ever increasing non-superconducting volume due to the increasing number of non-superconducting vortex cores, hence an overall lower number of Cooper pairs.

Here, we should note that strong applied field of $1 \mathrm{~T}$ likely may also assist in enhancement of spin polarisation in the LCMO layer to its complete saturation as can be seen in Fig. 3a). However, if the superconducting Cooper pairs play a significant role in suppressing the ability of spin polarisation within the double-exchange mechanism $[27,28]$ as proposed above, it is likely that the effect of the magnetisation saturation in the LCMO layer at the high field would play a secondary role.

For comparison, the MSLD profiles in the insets of Figure 5 have also been fitted with an alternative magnetic profile. For the low field measurements (Figure 5a, c), this profile is named 'Uniform M' that assumes a constant magnetisation up to the LCMO/STO interface. For high-field measurements (Figure 5b, d), this profile is named 'MD Layer' that assumes a magnetically dead layer in LCMO adjacent to the LCMO/STO interface. The vertical black stripes in the MSLD insets indicate that these alternate models only vary from the "Best Fit" close to the STO interface.

To reinforce the claim of the MD layer being present exclusively in low magnetic fields below the superconducting $T_{c}$, residual sum of squares were taken between the magnetic SLD models in the insets of Figure 5 ('Best fit' and 'Uniform M/MD layer') and the datapoints. This gives a statistical comparison between the quality of each fit, using the equation

$$
\chi=\sum_{i}\left[\mathrm{NSA}\left(q_{i}\right)-f\left(q_{i}\right)\right]^{2},
$$

where $\operatorname{NSA}\left(q_{i}\right)$ and $f\left(q_{i}\right)$ are the data points and fitted model at momentum transfer $q_{i}$ respectively. The smaller the $\chi$, the better the fit. Thus, from the $\chi$ values corresponding to each MSLD model in the insets of Figure 5, it is conclusive that a magnetically depleted layer is present below $T_{c}$ in low magnetic fields. It is because for the Best fit $\chi=1.093$, which is smaller than $\chi=1.622$ for the Uniform Fit (Figure 5a and c), and after subsequently applying a high magnetic field this depleted layer is restored: the Best Fit with $\chi=0.222$ is smaller than $\chi=0.420$ for the MD Layer Fit (Figs. 5b and d). 

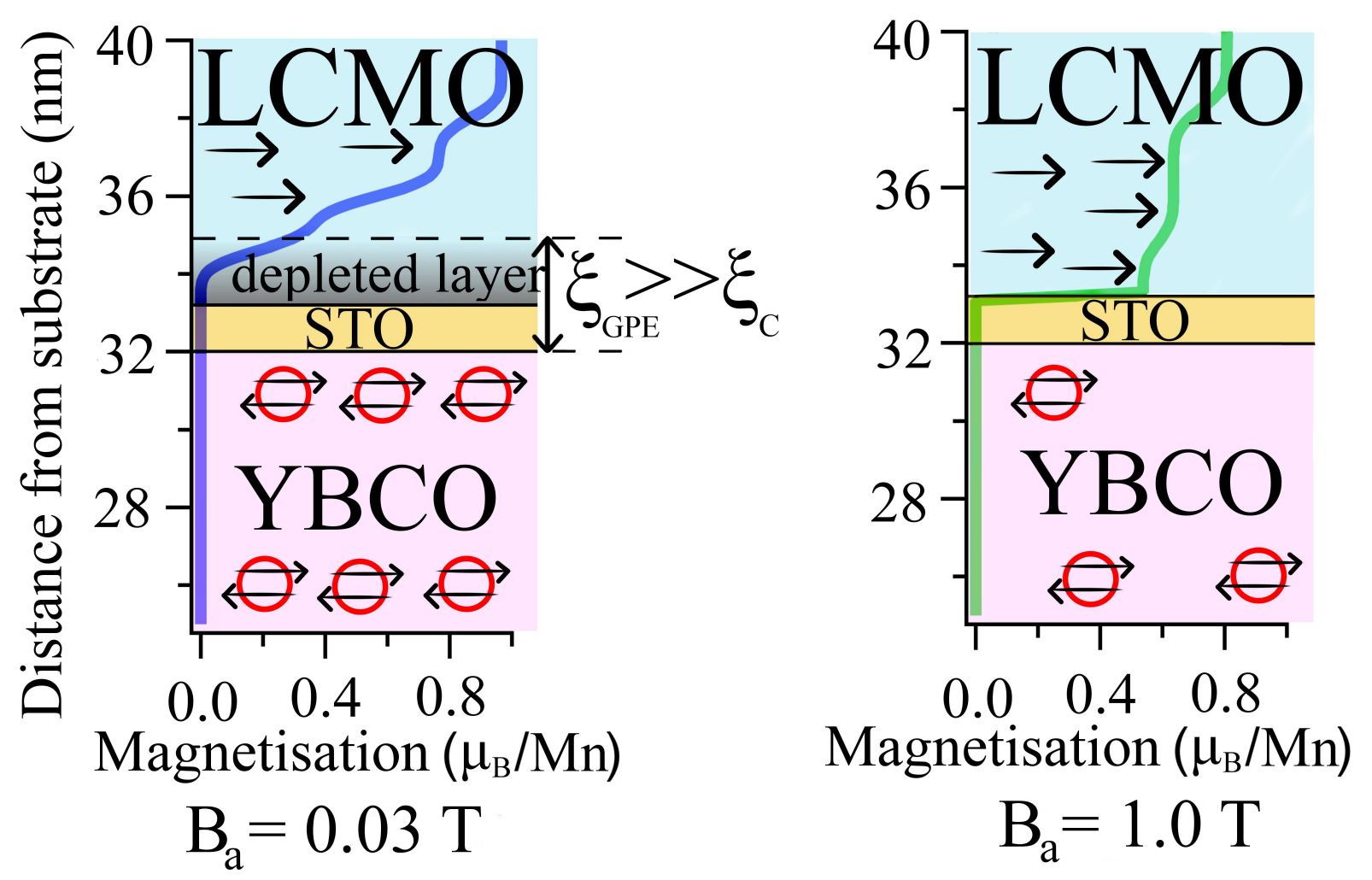

FIG. 6. Magnetisation profile at $60 \mathrm{~K}$ near the LCMO/STO interface in low (0.03 T) and high $(1 \mathrm{~T})$ field. At low field the length of the proximity effect $\xi_{\mathrm{GPE}}$ is significantly larger than the superconducting coherence length $\xi_{c}$.

\section{SUMMARY}

In this work, the magnetic field dependence of the MD layer in a YBCO $(30 \mathrm{~nm}) / \mathrm{STO}$ $(1.5 \mathrm{~nm}) / \mathrm{LCMO}(30 \mathrm{~nm})$ was investigated. The thickness of the MD layer within LCMO is identified to be $\sim 1.5 \mathrm{~nm}$, which is approximately an order of magnitude larger than the superconducting $c$-axis coherence length $\left(\xi_{c}=0.2-0.3 \mathrm{~nm}\right)$ for YBCO [29]. The total thickness of the STO interlayer and MD layer indicates that the length $\left(\xi_{\mathrm{GPE}}\right)$ of the ferromagnetic/superconducting proximity effect across the LCMO/STO/YBCO interfaces measurably extends over $\xi_{\mathrm{GPE}} \sim 10$ to $15 \xi_{c}$. When compared to the model corresponding to the dataset collected in $1 \mathrm{~T}$, this depleted layer is restored (see Figure 6). The large length of this proximity effect is reminiscent of a Giant Proximity Effect (GPE) that has been observed in Superconductor/Normal metal/Superconductor (SNS) trilayer sandwiches with sizeable supercurrent flowing through barriers of up to $\sim 100 \xi_{c}(20 \mathrm{~nm})$ thick [30]. 
A similar Josephson tunnelling through a $20 \mathrm{~nm}$ barrier for Superconductor/Ferromagnetic Insulator/Superconductor (S-FI-S) junctions was also found by applying a voltage across the superconducting electrodes [31]. The proximity effect facilitates Josephson tunnelling. Hence, in our system of a Superconductor/Insulator/Ferromagnetic Half-Metal (S-I-FHM) with a thin insulating layer, the magnetically depleted layer could be the result of GPE with the Cooper pair tunnelling through (or transported across) the insulator due to supercurrents arising in YBCO in response to the applied magnetic field. Once a Cooper pair has reached the LCMO layer, one (or both) electron(s) from the Cooper pairs becomes bound with an initially unpaired electron in the Mn $d$-orbital of LCMO, which is the origin of the double-exchange mechanism responsible for its ferromagnetism [27, 28], thereby suppressing it.

In conclusion, the experimental results obtained by XRR, XRD, VSM and PNR are conclusive that there is simultaneous ferromagnetic and superconducting ordering within the trilayer. The magnetically depleted layer has been found within LCMO layer adjacent to STO/LCMO interface in low magnetic fields, which is attributed to the proximity effect between superconducting and magnetic materials. The MD layer in LCMO is clearly shown to be triggered by the influence of superconductivity in YBCO across the STO interlayer. The overall proximity effect extends over the length of up to $\xi_{\mathrm{GPE}} \sim 15 \xi_{c}$. Notably, the results presented here provide convincing evidence through comparison between normalised spin asymmetry (NSA) data at low and high magnetic fields that the magnetically depleted layer can be restored under the application of a high magnetic field, which partially suppresses the superconductivity in YBCO. This may support the hypothesis that the MD layer is dependent on the density of superconducting Cooper pairs tunnelling (or being transported) from the YBCO through the STO into the LCMO over length scales significantly larger than the coherence length $\xi_{c}$. The fact that the MD layer can be reliably restored and controlled, indicates potential for future spintronic devices. 


\section{METHODS}

\section{A. Sample Growth}

The $\mathrm{YBCO}(30 \mathrm{~nm}) / \mathrm{STO}(1.5 \mathrm{~nm}) / \mathrm{LCMO}(30 \mathrm{~nm})$ trilayers were grown with pulsed laser deposition (PLD) on a Yttria-Stabilised Zirconia (YSZ) substrate as described in our previous work $[15,16]$. The PLD system uses a $248 \mathrm{~nm} \mathrm{KrF}$ laser with a fluence of $1-2 \mathrm{~J} \mathrm{~cm}^{-2}$. The pressure of the deposition chamber is maintained at $300 \mathrm{mTorr}$, and the samples are kept at $780^{\circ} \mathrm{C}$. The frequency of the laser is set to $4 \mathrm{~Hz}$ during deposition.

YSZ substrates have been chosen to substitute STO, in order to eliminate effects from the structural phase transition of STO which occurs below $105 \mathrm{~K}$, changing the crystal structure from cubic perovskite to tetragonal $[32,33]$. Although STO is well known to facilitate the best superconducting properties of YBCO thin films [16] due to the compatibility of their crystal lattice parameters, the structural distortion at $105 \mathrm{~K}$ creates crystallographic facets in the surface of the STO substrate, promoting off-specular scattering, which significantly restricts the quality of specular PNR methods. Similarly, Uribe-Laverde et al. used $\mathrm{La}_{0.3} \mathrm{Sr}_{0.7} \mathrm{Al}_{0.65} \mathrm{Ta}_{0.35} \mathrm{O}_{3}$ as a substrate to substitute STO [4].

\section{B. Measurement Techniques}

$\mathrm{X}$-ray reflectometry $(\mathrm{XRR})$ is utilised to determine the quality of the surfaces in the sample, the thickness of the material layers, and the roughness between material layers. This is done using the PANalytical Xpert PRO X-ray reflectometer using $\omega-2 \theta$ geometry. XRR data are fitted using Motofit [34]. X-ray diffraction (XRD) is performed on the same machine in diffraction mode to determine the quality of the epitaxial growth of the crystal structure of the materials used.

The SLD for X-rays and neutrons in a given material is a measure of the incident particle's ability to be scattered from the material. In a multilayered material, X-ray and neutron reflectometry allow to reconstruct the depth-dependence of the SLDs and over what distance the SLD transitions from one layer to the other which is a measure of the interfacial roughness [35].

PNR was performed at Australian Nuclear Science and Technology Organisation (ANSTO) in Sydney using PLATYPUS, the time-of-flight polarised neutron reflectometer [36, 37]. 
PNR is a non-destructive technique that allows the chemical and magnetic profiles to be determined in a sample with sub-nanometre depth resolution. The SLD profile corresponding to a given polarised neutron reflectivity pattern is comprised of a nuclear and magnetic component, $\rho_{ \pm}=\rho_{n}+\rho_{m}=\rho_{n} \pm C \sum_{i} N_{i} \mu_{i}$ where $\rho_{n}$ and $\rho_{m}$ is the nuclear and magnetic SLD, $C=2.645 \mathrm{fm} \mu_{B}^{-1}, N_{i}$ is the number density of the $i$ th atom, $\mu_{i}$ is the magnetic moment of the $i$ th atom in Bohr magnetons. The magnetic SLD is added or subtracted from the nuclear SLD depending on whether the spin of the incident neutron is parallel or anti-parallel to the magnetisation of the sample [38].

A Quantum Design Physical Properties Measurement System (PPMS) is used to measure the sample's magnetic and transport current properties using the Vibrating Sample Magnetometer (VSM) and resistivity measurement options, respectively. Magnetisation has been measured with the magnetic field applied in-plane to the trilayer, which is parallel to the surface of the sample. We should note that a certain degree of misalignment (of $\sim 5$ degrees) between the surface and the applied magnetic field should be expected for all the type of measurements.

Due to the irreversible magnetic nature of the YBCO type II superconductor, the magnetic history of measured samples has been reproduced for all measurements. During PNR (and PPMS) measurements, the sample is field-cooled to the desired temperature in the magnetic field of $0.03 \mathrm{~T}$ applied in-plane of the films in order to fully saturate magnetisation of LCMO before cooling it through the superconducting transition temperature $T_{c}$. Then, the magnetic field is tuned (or swept) to the desired strength.

The resistivity measurements have been carried out employing the standard 4-probe technique with $\mathrm{Au} / \mathrm{Pd}$ contact pads of about $100 \mathrm{~nm}$ thick deposited as a series of four stripes on the top of the LCMO layer. The $T_{c}$ of the $30 \mathrm{~nm}$ thick YBCO layer was determined as the onset of the superconducting transition measured at $100 \mu \mathrm{A}$ in zero magnetic field.

\section{ACKNOWLEDGEMENT}

This work is supported by the Australian Research Council via A. V. Pan's Discovery Project DP110100398, by the Faculty of Engineering and Information Sciences (University of Wollongong, Australia), by Australian Nuclear Science and Technology Organisation (ANSTO) Neutron Proposals (P5351 and P6169), by the Australian Government Research 
Training Program Awards, and the Australian Institute of Nuclear Science and Engineering (AINSE) Postgraduate Research Awards. O. H. C. Paull also acknowledges support from the AINSE Honours Scholarship stipend. X. Liu appreciates hospitality and support from ANSTO during his stay as an Asia-Oceania Neutron Scattering Association (AONSA) Young Research Fellow.

[1] Chakhalian, J., Freeland, J. W., Habermeier, H. -U., Cristiani, G., Khaliullin, G., van Veenendaal, M., Keimer, B., Orbital Reconstruction and Covalent Bonding at an Oxide Interface, Science 318, 1114 (2007).

[2] Franö, A., Blanco-Canosa, S., Schierle, E., Lu, Y., Wu, M., Bluschke, M., Minola, M., Christiani, G., Habermeier, H. -U., Logvenov, G. et al., Long-Range Charge-Density-Wave Proximity Effect at Cuprate/Manganite Interfaces, Nat. Mater. 15, 831 (2016).

[3] Satapathy, D. K., Uribe-Laverde, M. A., Marozau, I., Malik, V. K., Das, S., Wagner, Th., Marcelot, C., Stahn, J., Brück, S., Rühm, A. et al. Magnetic Proximity Effect in $\mathrm{YBa}_{2} \mathrm{Cu}_{3} \mathrm{O}_{7} / \mathrm{LaMnO}_{3-\delta}$ Superlattices. Phys. Rev. Lett. 108, 197201 (2012).

[4] Uribe-Laverde, M. A., Satapathy, D. K., Marozau, I., Malik, V. K., Das, S., Sen, K., Stahn, J., Rühm, A., Kim, J.-H., Keller, T. et al. Depth Profile of the Ferromagnetic Order in a $\mathrm{YBa}_{2} \mathrm{Cu}_{3} \mathrm{O}_{7} / \mathrm{La}_{2 / 3} \mathrm{Ca}_{1 / 3} \mathrm{MnO}_{3}$ superlattice on a LSAT substrate: A Polarized Neutron Reflectometry Study, Phys. Rev. B 87, 115105 (2013).

[5] Hoppler, J., Stahn, J., Niedermayer, Ch., Malik, V. K., Bouyanfif, H., Drew, A. J., Rössle, M., Buzdin, A., Cristiani, G., Habermeier, H.-U. et al. Giant Superconductivity-Induced Modulation of the Ferromagnetic Magnetization in a Cuprate/Manganite Superlattice, Nat. Mater. 8, 315 (2009).

[6] Luo, W., Pennycook, S. J., Pantelides, S. T., Magnetic "Dead" Layer at a Complex Oxide Interface, Phys. Rev. Lett. 101, 247204 (2008).

[7] Hwang, H. Y., Iwasa, Y., Kawasaki, M., Keimer, B., Nagaosa, N., and Tokura, Y., Emergent Phenomena at Oxide Interfaces, Nat. Mater. 11, 103 (2012).

[8] Fedoseev, S. A., Investigation of Superconducting Thin Films and Multilayered Structures for Electronic Applications, PhD Thesis, University of Wollongong, Australia (2014). 
[9] Yonamine, A. H., Fedoseev, S. A., dos Santos, D. I., and Pan, A. V., Magnetic Properties of YBCO/LCMO Superlattices With And Without STO Interlayers, Adv. Mater. Res. 975, 101 (2014).

[10] Prajapat, C. L., Singh, S., Paul, A., Bhattacharya, D., Singh, M. R., Mattauch, S., Ravikumara, G., \& Basu, S. Superconductivity-Induced Magnetization Depletion in a Ferromagnet Through an Insulator in a FerromagnetInsulatorSuperconductor Hybrid Oxide Heterostructure. Nanoscale 8, 10188 (2016).

[11] C. L. Prajapat, S. Singh, D. Bhattacharya, G. Ravikumar, S. Basu, S. Mattauch, J.-G. Zheng, T. Aoki, and A. Paul, Sci. Rep., 8:3732 (2018).

[12] Fullerton, E. E., Guimpel, J., Nakamura, O., and Schuller, I. K., Structure of High-T $\mathrm{Su}^{-}$ perlattices, Phys. Rev. Lett. 69, 2859 (1992).

[13] Zheng, X.-Y., Lowndes, D. H., Zhu, S., Budai, J. D., and Warmack, R. J. Early Stages of $\mathrm{YBa}_{2} \mathrm{Cu}_{3} \mathrm{O}_{7-\delta}$ Epitaxial Growth on $\mathrm{MgO}$ and $\mathrm{SrTiO}_{3}$, Phys. Rev. B 45, 7584 (1992).

[14] Pan, A. V., Pysarenko, S. V., Wexler, D., Rubanov, S., and Dou, S. X., Multilayering and Ag-Doping for Properties and Performance Enhancement in $\mathrm{YBa}_{2} \mathrm{Cu}_{3} \mathrm{O}_{7}$ Films, IEEE Trans. Appl. Supercond. 17, 3585 (2007).

[15] Fedoseev, S. A., Pan, A. V., Rubanov, S., Golovchanskiy, I. A., and Shcherbakova, O. V., Large, Controllable Spikes of Magnetoresistance in $\mathrm{La}_{2 / 3} \mathrm{Ca}_{1 / 3} \mathrm{MnO}_{3} / \mathrm{SrTiO}_{3}$ Superlattices, ACS Nano 7, 286 (2013).

[16] Pan, A. V., Pysarenko, S., and Dou, S. X., Drastic Improvement of Surface Structure and Current-carrying Ability in $\mathrm{YBa}_{2} \mathrm{Cu}_{3} \mathrm{O}_{7}$ Films by Introducing Multilayered Structure, Appl. Phys. Lett. 88, 232506 (2006).

[17] O. V. Shcherbakova, A. V. Pan, S. K. Gorman, S. A. Fedoseev, I. A. Golovchanskiy, and S. $\mathrm{X}$. Dou, Inhomogeneities in $\mathrm{YBa}_{2} \mathrm{Cu}_{3} \mathrm{O}_{7}$ thin films with reduced thickness, Physica $C \mathbf{4 7 9}$, $102(2012)$.

[18] M. A. Sillanpää, T. T. Heikkilä, R. K. Lindell, and P. J. Hakonen, Inverse proximity effect in superconductors near ferromagnetic material, EPL 56, 590 (2001).

[19] Y. Kalcheim, O. Millo, A. Di Bernardo, A. Pal, and J. W. A. Robinson, Inverse proximity effect at superconductor-ferromagnet interfaces: Evidence for induced triplet pairing in the superconductor, Phys. Rev. B 92, 060501(R) (2015). 
[20] Pan, A. V., Zhou, S., Liu, H., and Dou, S., Direct visualization of iron sheath shielding effects in $\mathrm{MgB}_{2}$ superconducting wires, Supercond. Sci. Technol. 16, L33 (2003).

[21] Pan, A. V. and Dou, S., Overcritical State in Superconducting Round Wires Sheathed by Iron, J. Appl. Phys. 96, 1146 (2004).

[22] "Simulreflec", Program and description available from http://wwwllb.cea.fr/prism/programs/simulreflec/simulreflec.html

[23] Spurgeon, S. R., Balachandran, P. V., Kepaptsoglou, D. M., Damodaran, A. R., Karthik, J., Nejati, S., Jones, L., Ambaye, H., Lauter, V., Ramasse, Q. M. et al., Polarization ScreeningInduced Magnetic Phase Gradients at Complex Oxide Interfaces, Nat. Commun. 6, 6735 (2015).

[24] Kawashima, K., Logvenov, G., Christiani, G., Habermeier, H.-U., Interrelation of Epitaxial Strain and Oxygen Deficiency in $\mathrm{La}_{0.7} \mathrm{Ca}_{0.3} \mathrm{MnO}_{3-\delta}$ Thin Films, J. Magn. Magn. Mater. 378, 539 (2015).

[25] Golovchanskiy, I. A., Pan, A. V., Fedoseev, S. A., Higgins, M. Significant Tunability of Thin Film Functionalities Enabled by Manipulating Magnetic and Structural Nano-Domains, Appl. Surf. Sci. 311, 549 (2014).

[26] Monclús, M. A., Karlik, M., Callisti, M., Frutos, E., Llorca, J., Polcar, T., \& MolinaAldareguía, J. M. Microstructure and Mechanical Properties of Physical Vapor Deposited $\mathrm{Cu} / \mathrm{W}$ Nanoscale Multilayers: Influence of Layer Thickness and Temperature. Thin Solid Films 571, 275 (2014).

[27] C. Zener, Interaction between the $d$-Shells in the Transition Metals. II. Ferromagnetic Compounds of Manganese with Perovskite Structure, Phys. Rev. 82, 403 (1951).

[28] P.-G. De Gennes, Effect of Double Exchange in Magnetic Crystals, Phys. Rev. 118, 141 (1960).

[29] Blatter, G., Feigel'man, M. V., Geshkenbein, V. B., Larkin, A. I., and Vinokur, V. M., Vortices in High-Temperature Superconductors, Rev. Mod. Phys. 66, 1125 (1994).

[30] Bozovic, I., Logvenov, G., Verhoeven, M. A. J., Caputo, P., Goldobin, E., and Beasley, M. R. Giant Proximity Effect in Cuprate Superconductors, Phys. Rev. Lett. 93, 157002 (2004).

[31] Golod, T., Rydh, A., Krasnov, V. M., Marozau, I., Uribe-Laverde, M. A., Satapathy, D. K., Wagner, Th., \& Bernhard, C. High Bias Anomaly in $\mathrm{YBa}_{2} \mathrm{Cu}_{3} \mathrm{O}_{7-x} / \mathrm{LaMnO}_{3+\delta} / \mathrm{YBa}_{2} \mathrm{Cu}_{3} \mathrm{O}_{7-x} \quad$ Superconductor/Ferromagnetic Insulator/Superconductor Junctions: Evidence for a Long-Range Superconducting Proximity 
Effect Through the Conduction Band of a Ferromagnetic Insulator, Phys. Rev. B 87, 134520 (2013).

[32] He, F., Wells, B. O., Shapiro, S. M., Zimmermann, M. V., Clark, A., and Xi, X. X., Anomalous Phase Transition in Strained $\mathrm{SrTiO}_{3}$ Thin Films, Appl. Phys. Lett. 83, 123 (2003).

[33] Hoppler, J., Fritzsche, H., Malik, V. K., Stahn, J., Cristiani, G., Habermeier, H.-U., Rössle, M., Honolka, J., Enders, A., \& Bernhard, C. Polarized Neutron Reflectometry Study of the Magnetization Reversal Process in $\mathrm{YBa}_{2} \mathrm{Cu}_{3} \mathrm{O}_{7} / \mathrm{La}_{2 / 3} \mathrm{Ca}_{1 / 3} \mathrm{MnO}_{3}$ Superlattices Grown on $\mathrm{SrTiO}_{3}$ Substrates, Phys. Rev. B 82, 174439 (2010).

[34] Nelson, A. Co-Refinement of Multiple-Contrast Neutron/X-ray Reflectivity Data Using MOTOFit J. Appl. Crystallogr. 39, 273 (2006).

[35] Sivia, D. S. Elastic Scattering in Elementary Scattering Theory: For X-ray and Neutron Users (Oxford Univ. Press, Oxford, 2011)

[36] James, M., Nelson, A., Holt, S., Saerbeck, T., Hamilton, W., \& Klose, F. The Multipurpose Time-of-Flight Neutron Reflectometer "Platypus at Australia's OPAL Reactor, Nucl. Instrum. Methods Phys. Res., Sect. A 632, 112 (2011).

[37] Saerbeck, T., Klose, F., Le Brun, A. P., Füzi, J., Brule, A., Nelson, A., Holt, S. A., and James, M., Polarization "Down Under": The polarized time-of-flight neutron reflectometer PLATYPUS, Rev. Sci. Instrum. 83, 081301 (2012).

[38] Fitzsimmons, M. R., Schuller, I. K., Neutron Scattering-The Key Characterization Tool for Nanostructured Magnetic Materials, J. Magn. Magn. Mater. 350, 199208 (2014). 
A giant ferromagnetic/superconducting proximity effect is demonstrated to be controllable by an external magnetic field.

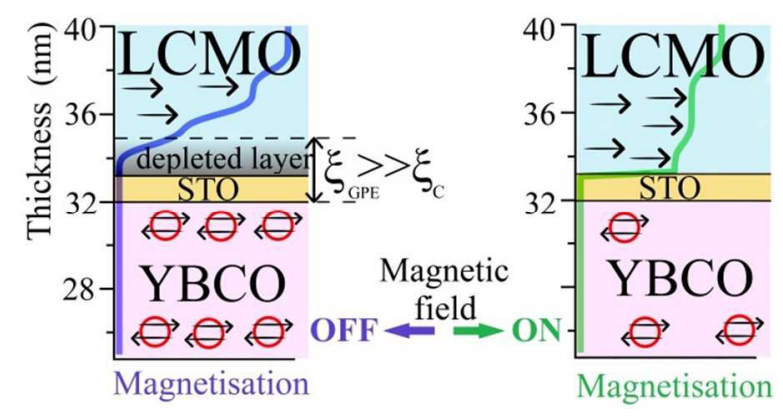

